\title{
Local and Global Isotropy Analysis of Mobile Robots with Three Active Caster Wheels
}

\author{
Sungbok Kim and Sanghyup Lee \\ Department of Digital Information Engineering \\ Hankuk University of Foreign Studies
}

Korea

\section{Introduction}

In the near future, personal service robots are expected to come into human daily life as supporters in education, leisure, house care, health care, and so on. Most of them are built on an omnidirectional mobile robot so as to navigate in an environment restricted in space and cluttered with obstacles. Caster wheels have been chosen for the development of an omnidirectional mobile robot, as reported in (Holmberg, 2000), among several omnidirectional wheel mechanisms, including universal wheels, Swedish wheels, orthogonal wheels, and ball wheels. This is because caster wheels can operate without additional peripheral rollers or support structure, and maintain good performance even though payload or ground condition changes.

There have been several works on the kinematics of a caster wheeled omnidirectional mobile robot (COMR), including the kinematic modeling, the design and control, the isotropy analysis, as reported in (Holmberg, 2000; Muir \& Neuman, 1987; Campion et al., 1996; Kim \& Kim, 2004; Park et al., 2002; Kim \& Moon, 2005; Oetomo et al., 2005; Kim \& Jung, 2007). Previous isotropy analysis, as reported in (Kim \& Kim, 2004; Park et al., 2002; Kim \& Moon, 2005; Oetomo et al., 2005), has been made only for a COMR in which the steering link offset is equal to the wheel radius, except our recent work, as reported in (Kim \& Jung, 2007). It was found that such a restriction is necessary to have globally optimal isotropic characteristics of a COMR, as reported in (Park et al., 2002; Kim \& Moon, 2005; Oetomo et al., 2005). Nevertheless, many practical COMR's in use take advantage of the steering link offset which is different from the wheel radius, mainly for improved tipover stability, as reported in (McGhee \& Frank, 1968; Papadopoulos \& Rey, 1996). The tipover stability becomes critical when a COMR makes a rapid turn or sudden external forces are applied to a COMR.

The accuracy of the velocity kinematics of a robotic system depends on the condition number of the Jacobian matrix involved. Based on the Euclidean norm, the condition number of a matrix can be defined as the ratio of the largest to smallest singular values of the matrix, as reported in (Strang, 1988), whose value ranges from unity to infinity. For a given linear system $\mathbf{A x}=\mathbf{b}$, the condition number of $\mathbf{A}$ represents the amplification of the 
relative error of $\mathbf{x}$, given by $\|\delta \mathbf{x}\| /\|\mathbf{x}\|$, with respect to the relative error of $\mathbf{b}$, given by $\|\delta \mathbf{b}\| /\|\mathbf{b}\|$. Smaller condition numbers are preferred to larger condition numbers with regard to error amplification in solving $\mathbf{A x}=\mathbf{b}$. Furthermore, the condition number equal to unity is the best situation that can be achieved when a robotic system is said to be in isotropy.

Since the Jacobian matrix of a COMR is a function of caster wheel configurations, the condition number is subject to change during task execution. For reduction in aforementioned error amplification, it is important to prevent a COMR away from the isotropy or keep a COMR close to the isotropy, as much as possible. In the light of this, this paper aims to investigate the local and global isotropic characteristics of a COMR. The isotropic configurations of a COMR which can be identified through the local isotropy analysis can be used as a reference in trajectory planning to avoid excessive error amplification throughout task execution. On the other hand, the design parameter of a COMR, such as wheel radius and steering link offset, can be optimized for the global isotropic characteristics by minimizing the averaged value of the condition number over the whole configuration space.

The purpose of this paper is to present both local and global isotropy analysis of a fully actuated COMR with the steering link offset different from the wheel radius. This paper is organized as follows. Based on the kinematic model, Section 2 derives the necessary and sufficient conditions for the isotropy of a COMR. Section 3 identifies four different sets of all possible isotropic configurations, along with the isotropic steering link offsets and the isotropic characteristic lengths. Using the local isotropy index, Section 4 examines the number of the isotropic configurations and the role of the isotropic characteristic length. Using global isotropy index, Section 5 determines the optimal characteristic length and the optimal steering link offset for maximal global isotropy. Finally, the conclusion is made in Section 6.

\section{Isotropy conditions}

Consider a COMR with three identical caster wheels attached to a regular triangular platform moving on the $x y$-plane, as shown in Fig. 1 . Let $l$ be the side length of the platform; let $d(\geq 0)$ and $r(>0)$ be the steering link offset and the wheel radius, respectively; let $\varphi_{i}$ and $\theta_{i}$ be the steering and the rotating angles, respectively; let $\mathbf{u}_{i}$ and $\mathbf{v}_{i}$ be the orthogonal unit vectors along the steering link and the wheel axis, respectively, such that $\mathbf{u}_{i}=\left[-\cos \varphi_{i}-\sin \varphi_{i}\right]^{t}$ and $\mathbf{v}_{i}=\left[-\sin \varphi_{i} \cos \varphi_{i}\right]^{t}$; let $\mathbf{p}_{i}$ be the vector from the center of the platform to the center of the wheel, and $\mathbf{q}_{i}$ be the rotation of $\mathbf{p}_{i}$ by $90^{\circ}$ counterclockwise. For each wheel, it is assumed that the steering link offset can be different from the wheel radius, that is, $d \neq r$.

With the introduction of the characteristic length, as reported in (Strang, 1988), L(>0), the kinematic model of a COMR under full actuation is obtained by

$$
\mathrm{A} \dot{x}=\mathrm{B} \dot{\Theta}
$$


where $\dot{\mathbf{x}}=[\mathbf{v} L \omega]^{t} \in \mathbf{R}^{3 \times 1}$ is the task velocity vector, and $\dot{\Theta}=\left[\dot{\theta}_{1} \dot{\theta}_{2} \dot{\theta}_{3} \dot{\varphi}_{1} \dot{\varphi}_{2} \dot{\varphi}_{3}\right]^{t} \in \mathbf{R}^{6 \times 1}$ is the joint velocity vector, and

$$
\begin{gathered}
\mathbf{A}=\left[\begin{array}{llll}
\mathbf{u}_{1}{ }^{t} & \frac{1}{L} & \mathbf{u}_{1}{ }^{t} & \mathbf{q}_{1} \\
\mathbf{u}_{2}{ }^{t} & \frac{1}{L} & \mathbf{u}_{2}{ }^{t} & \mathbf{q}_{2} \\
\mathbf{u}_{3}{ }^{t} & \frac{1}{L} & \mathbf{u}_{3}{ }^{t} & \mathbf{q}_{3} \\
\mathbf{v}_{1}{ }^{t} & \frac{1}{L} & \mathbf{v}_{1}{ }^{t} & \mathbf{q}_{1} \\
\mathbf{v}_{2}{ }^{t} & \frac{1}{L} & \mathbf{v}_{2}{ }^{t} & \mathbf{q}_{2} \\
\mathbf{v}_{3}{ }^{t} & \frac{1}{L} & \mathbf{v}_{3}{ }^{t} & \mathbf{q}_{3}
\end{array}\right] \in \mathbf{R}^{6 \times 3} \\
\mathbf{B}=\left[\begin{array}{cc}
r \mathbf{I}_{3} & 0_{3} \\
0_{3} & d \mathbf{I}_{3}
\end{array}\right] \in \mathbf{R}^{6 \times 6}
\end{gathered}
$$

are the Jacobian matrices. Notice that the introduction of $L$ makes all three columns of $\mathbf{A}$ to be consistent in physical unit.

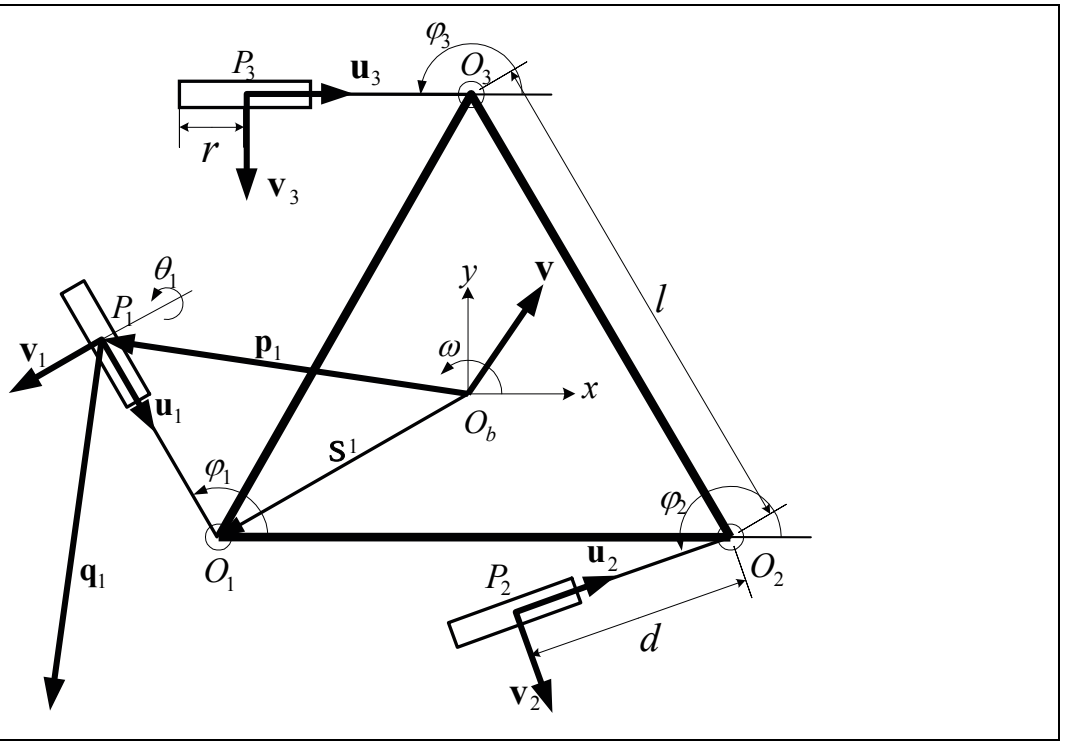

Fig. 1. A caster wheeled omnidirectional mobile robot

Now, from (1), the necessary and sufficient condition for the kinematic isotropy of a COMR can be expressed as

$$
\mathbf{Z}^{t} \mathbf{Z}=\sigma \mathbf{I}_{3}
$$


where

$$
\begin{gathered}
\mathbf{Z}=\mathbf{B}^{-1} \mathbf{A} \\
\sigma=\frac{3}{2}\left(\frac{1}{r^{2}}+\frac{1}{d^{2}}\right)
\end{gathered}
$$

Using (2), (3), (5), and (6), from (4), the three isotropy conditions of a COMR can be obtained as follows:

$$
\begin{gathered}
\sum_{i=1}^{3}\left[\mu\left(\mathbf{u}_{i} \mathbf{u}_{i}{ }^{t}\right)+\left(\mathbf{v}_{i} \mathbf{v}_{i}{ }^{t}\right)\right]=\frac{3}{2}(\mu+1) \mathbf{I}_{2} \\
\sum_{i=1}^{3}\left[\mu\left(\mathbf{u}_{i}{ }^{t} \mathbf{q}_{i}\right) \mathbf{u}_{i}+\left(\mathbf{v}_{i}{ }^{t} \mathbf{q}_{i}\right) \mathbf{v}_{i}\right]=\mathbf{0} \\
\frac{1}{L^{2}} \sum_{i=1}^{3}\left[\mu\left(\mathbf{u}_{i}{ }^{t} \mathbf{q}_{i}\right)^{2}+\left(\mathbf{v}_{i}{ }^{t} \mathbf{q}_{i}\right)^{2}\right]=\frac{3}{2}(\mu+1)
\end{gathered}
$$

where

$$
\mu=\left(\frac{d}{r}\right)^{2}>0
$$

which represents the square of the ratio of the steering link offset $d$ to the wheel radius $r$. Note that $\mu=1$ corresponds to the case of the steering link offset $d$ equal to the wheel radius $r$, as reported in (Kim \& Kim, 2004).

\section{Isotropic configurations}

The first and the second isotropy conditions, given by (7) and (8), are a function of the steering joint angles, $\left(\varphi_{1}, \varphi_{2}, \varphi_{3}\right)$, from which the isotropic configurations, denoted by $\Theta_{\text {iso }}$, can be identified. For a given wheel radius $r$, the specific value of steering link offset, called as the isotropic steering link offset, $d_{\text {iso, }}$, is required for the isotropy of a COMR. With the isotropic configuration known, the third isotropy condition, given by (9), determines the specific value of the characteristic length, called the isotropic characteristic length, $L_{\mathrm{iso}}$, is required for the isotropy of a COMR. The detailed procedure to obtain the isotropic configurations $\Theta_{\text {iso }}$, the isotropic steering link offset $d_{\text {iso }}$, and the isotropic characteristic length $L_{\text {iso }}$ can be found our previous work, as reported in (Kim \& Jung, 2007).

All possible isotropic configurations $\Theta_{\text {iso }}$ of a COMR can be categorized into four different sets according to the restriction imposed on the isotropic steering link offset $d_{\text {iso }}$, for a given 


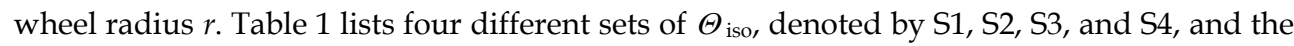
corresponding value of $d_{\text {iso }}$.

\begin{tabular}{|c|c|c|c|}
\hline Set & $\Theta_{\text {iso }}$ & $d_{\text {iso }}$ & $L_{\text {iso }}$ \\
\hline S1 & $\begin{array}{c}\varphi_{1} \\
\varphi_{1}+\frac{2 \pi}{3} \\
\varphi_{1}-\frac{2 \pi}{3}\end{array}$ & $\begin{array}{l}\quad \text { No } \\
\text { restriction } \\
\mu=\left(\frac{d}{r}\right)^{2}\end{array}$ & $\sqrt{\frac{2}{\mu+1}\left\{\left(\frac{1}{\sqrt{3}} \sin \left(\varphi_{1}-\frac{\pi}{6}\right)\right)^{2} \mu+\left(d-\frac{1}{\sqrt{3}} \cos \left(\varphi_{1}-\frac{\pi}{6}\right)^{2}\right\}\right.}$ \\
\hline S2 & $\begin{array}{c}\varphi_{1} \\
\varphi_{1}-\frac{2 \pi}{3} \\
\varphi_{1}+\frac{2 \pi}{3}\end{array}$ & $r$ & $\sqrt{d_{\text {iso }}{ }^{2}+\frac{1}{3}}$ \\
\hline S3 & $\begin{array}{l}\left(\frac{\pi}{6}, \frac{\pi}{2},-\frac{\pi}{6}\right), \\
\left(-\frac{\pi}{6},-\frac{5 \pi}{6},-\frac{\pi}{2}\right), \\
\left(\frac{\pi}{2}, \frac{5 \pi}{6},-\frac{5 \pi}{6}\right)\end{array}$ & $\begin{array}{r}\sqrt{r^{2}}+\frac{4}{3} r^{4} \\
-\frac{2}{\sqrt{3}} r^{2}\end{array}$ & $\frac{1}{\sqrt{1-\frac{2}{\sqrt{3}} d_{\text {iso }}}}\left(\frac{1}{\sqrt{3}}-d_{\text {iso }}\right)$ \\
\hline S4 & $\begin{array}{c}\left(-\frac{5 \pi}{6},-\frac{\pi}{2}, \frac{5 \pi}{6}\right), \\
\left(\frac{5 \pi}{6}, \frac{\pi}{6}, \frac{\pi}{2}\right), \\
\left(-\frac{\pi}{2},-\frac{\pi}{6}, \frac{\pi}{6}\right)\end{array}$ & $\begin{array}{l}\sqrt{r^{2}+\frac{4}{3} r^{4}} \\
+\frac{2}{\sqrt{3}} r^{2}\end{array}$ & $\frac{1}{\sqrt{1+\frac{2}{\sqrt{3}} d_{\text {iso }}}}\left(\frac{1}{\sqrt{3}}+d_{\text {iso }}\right)$ \\
\hline
\end{tabular}

Table 1. Four different sets of all isotropic configurations

It should be noted that $\mathrm{S} 1$ places no restriction on $d_{\text {iso }}$, unlike the other three sets, $\mathrm{S} 2, \mathrm{~S} 3$, and S4. Fig. 2 illustrates four different sets of $\Theta_{\text {iso, }}$ characterized by the tuple of $\left(\mathbf{u}_{1}, \mathbf{u}_{2}, \mathbf{u}_{3}\right)$. It is interesting to observe that there exist certain geometrical symmetries among four sets: the symmetry between S1 and S2, shown in Fig. 2a) and 2b), and the symmetry between S3 and S4, shown in Fig. 2c) and 2d).

Once the isotropic configuration has been identified under the conditions of (7) and (8), the isotropic characteristic length $L_{\text {iso }}$ can be determined under the condition of (9). For four different sets of the isotropic configurations, the expression of $L_{\text {iso }}$ can be elaborated as 
listed in Table 1. Note that the isotropy of a COMR cannot be achieved unless the characteristic length is chosen as the isotropic characteristic length, that is, $L=L_{\text {iso }}$.

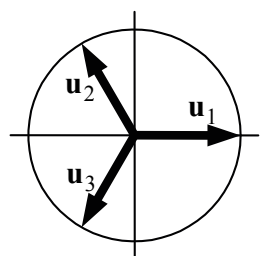

a)
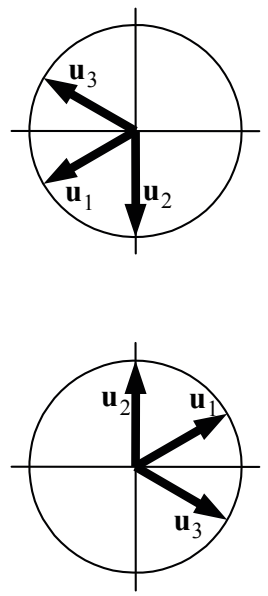

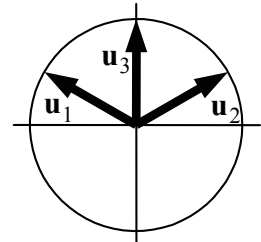

c)

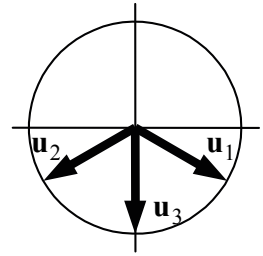

d)

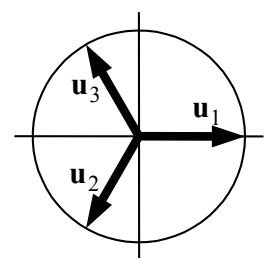

b)
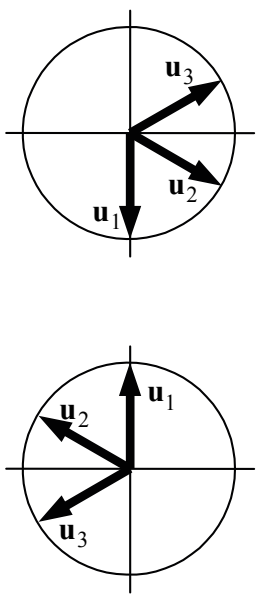

Fig. 2. Four different sets of the isotropic configurations: a) S1 with $\varphi_{1}=\pi$, b) S2 with $\left.\varphi_{1}=\pi, \mathrm{c}\right) \mathrm{S} 3$ and d) S4

\section{Local isotropy analysis}

Let $\lambda_{i}, i=1,2,3$, be the eigenvalues of $\mathbf{Z}^{t} \mathbf{Z}$, whose square roots are the same as the singular values of $\mathbf{Z}$. We define the local isotropy index of a COMR, denoted by $\sigma$, as

$$
0.0 \leq \sigma=\sqrt{\frac{\min \lambda_{i}}{\max \lambda_{i}}} \leq 1.0
$$

whose value ranges between 0 and 1 . Note that the local isotropy index $\sigma$ is the inverse of the well-known condition number of $\mathbf{Z}$. In general, $\sigma$ is a function of the wheel configuration $\Theta=\left(\varphi_{1}, \varphi_{2}, \varphi_{3}\right)$, the characteristic length $L$, the wheel radius $r$, and the steering link offset $d$ : 


$$
\sigma=\sigma(\Theta, L, r, d)
$$

To examine the isotropic characteristics of a COMR, extensive simulation has been performed for various combinations of characteristic length $L$, the wheel radius $r$, and the steering link offset $d$. However, we present only the simulation results obtained from two different situations, denoted by SM1 and SM3, for which the values of the key parameters, including $r, d_{\text {iso }}, \Theta_{\text {iso, }}$ and $L_{\text {iso }}$, are listed in Table 2. Note that all the values of $r, d$, and $L$ represent the relative scales to the platform side length $l$, which is assumed to be unity, that is, $l=1.0[\mathrm{~m}]$.

\begin{tabular}{|c|c|c|c|c|}
\hline Situation & $r$ & $d_{\text {iso }}$ & $\Theta_{\text {iso }}$ & $L_{\text {iso }}$ \\
\hline SM1 & 0.2 & 0.2 & $\left(\frac{\pi}{6}, \frac{5 \pi}{6},-\frac{\pi}{2}\right)$ & 0.3774 \\
\hline SM3 & 0.2 & 0.1591 & $\left(\frac{\pi}{6}, \frac{\pi}{2},-\frac{\pi}{6}\right)$ & 0.4629 \\
\hline
\end{tabular}

Table 2. Simulation environment

First, let us examine how the value of the local isotropy index $\sigma$ changes over the entire configuration space $\Theta=\left(\varphi_{1}, \varphi_{2}, \varphi_{3}\right)$. With the values of $r, d_{\text {iso }}$, and $L_{\text {iso }}$ given in Table 2, Fig. 3 shows the plots of $\sigma\left(\varphi_{1}=\pi / 6\right)$ for $-\pi \leq \varphi_{2}, \varphi_{3} \leq \pi$ in the cases of SM1 and SM3.

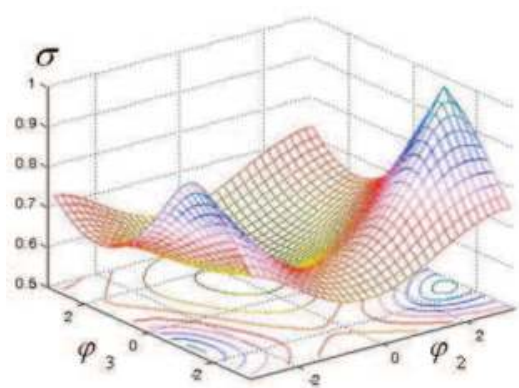

a)

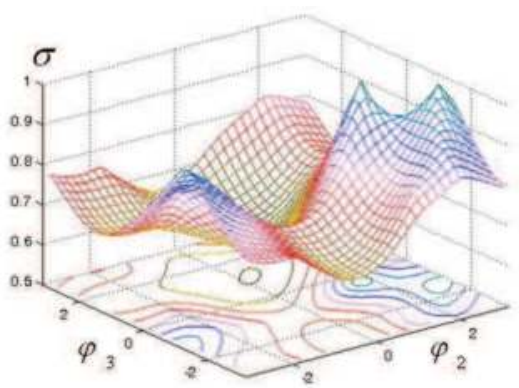

b)

Fig. 3. The plots of $\sigma\left(\varphi_{1}=\pi / 6\right)$ for $\left.-\pi \leq \varphi_{2}, \varphi_{3} \leq \pi: a\right)$ SM1 and b) SM3

The ranges of $\sigma$ are obtained as $0.5336 \leq \sigma\left(\varphi_{1}=\pi / 6\right) \leq 1.0$ for SM1 and $0.5972 \leq \sigma\left(\varphi_{1}=\pi / 6\right) \leq 1.0$ for SM3. For both cases, it can be observed that the value of $\sigma$ changes significantly depending on the wheel configurations and also that the isotropic configurations with $\sigma=1.0$ appear as the result of $d=d_{\text {iso }}$ and $L=L_{\text {iso }}$. Note that SM1 has a single isotropic configuration, $(\pi / 6,5 \pi / 6,-\pi / 2)$ which belongs to S1, whereas SM3 has 
two isotropic configurations: $(\pi / 6, \pi / 2,-\pi / 6)$ which belongs to S3 and $(\pi / 6,5 \pi / 6,-\pi / 2)$ which belongs to both S1 and S3.

Next, for a given isotropic configuration, let us examine how the choice of the characteristic length $L$ affects the values of the local isotropy index $\sigma$. With the values of $r, d_{\text {iso }}$ and $\Theta_{\text {iso }}$ given in Table 2, Fig. 4 shows the plots of $\sigma\left(\Theta_{\text {iso }}\right)$ for $0<L \leq 1.0$, in the cases of SM1 and SM3. For both cases, it can be observed that the value of $\sigma$ decreases significantly as the choice of $L$ is away from the isotropic characteristic length $L_{\text {iso }}: L_{\text {iso }}=0.377$ for SM1, and $L_{\text {iso }}=0.463$ for SM3. This demonstrates the importance of $L=L_{\text {iso }}$ for the isotropy of a COMR.

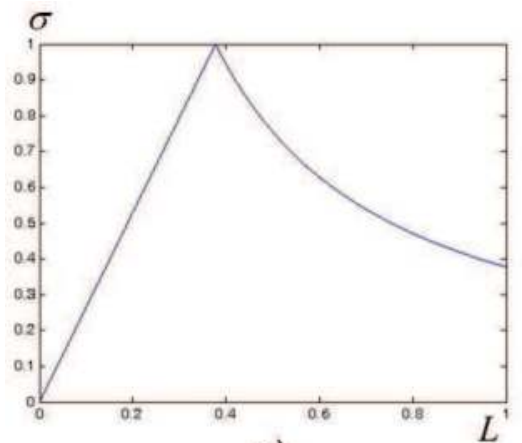

a)

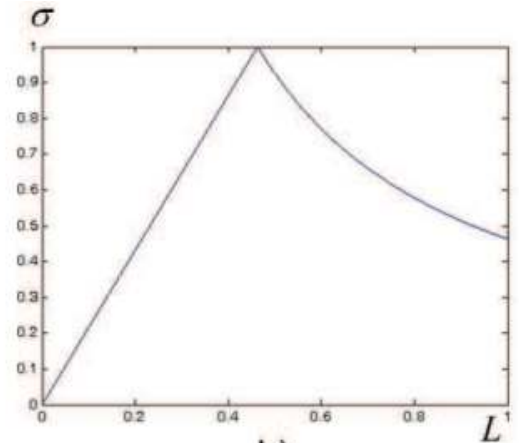

b)

Fig. 4. The plots of $\sigma\left(\Theta_{\text {iso }}\right)$ for $0<L \leq 1.0:$ a) SM1 and b) SM3

\section{Global isotropy analysis}

The local isotropy index represents the local isotropic characteristics of a COMR at a specific instance of the wheel configurations. To characterize the global isotropic characteristics of a COMR, we define the global isotropy index of a COMR, denoted by $\bar{\sigma}$, as the average of the local isotropy index $\sigma$ taken over the entire configuration space, $-\pi \leq \varphi_{1}, \varphi_{2}, \varphi_{3} \leq \pi$. Now, $\bar{\sigma}$ is a function of the characteristic length $L$, the wheel radius $r$, and the steering link offset $d$ :

$$
\bar{\sigma}=\bar{\sigma}(L, r, d)
$$

Let us examine how the choice of the characteristic length $L$ affects the values of the global isotropy index $\bar{\sigma}$. With the values of $r$ and $d_{\text {iso }}$ given in Table 2, Fig. 5 shows the plots of $\bar{\sigma}$ for $0<L \leq 1.0$, in the cases of SM1 and SM3. For both cases, it can be observed that the value of $\bar{\sigma}$ reaches its maximum, which is called as the optimal global isotropy index, $\bar{\sigma}_{\max }$, at the specific value of $L$, which is called as the optimal characteristic length, $L_{\mathrm{opt}}$ : $\bar{\sigma}_{\max }=0.8017$ at $L_{\mathrm{opt}}=0.614$ for SM1, and $\bar{\sigma}_{\max }=0.7538$ at $L_{\mathrm{opt}}=0.588$ for SM3. 


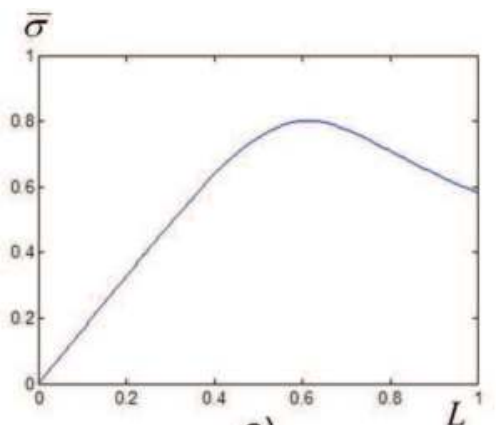

a)

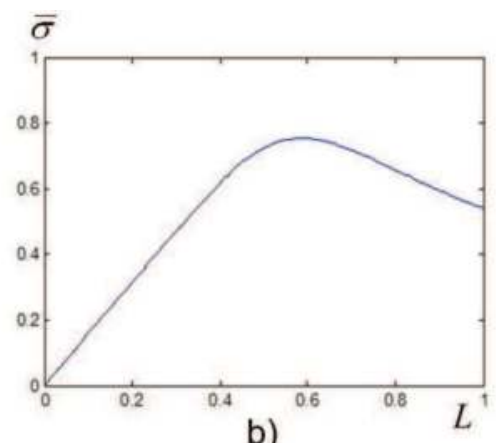

b)

Fig. 5. The plots of $\bar{\sigma}$ for $0<L \leq 1.0:$ a) SM1 and b) SM3

Next, let us examine how the ratio of the steering link offset $d$ to the wheel radius $r$ affects the values of the optimal global isotropy index $\bar{\sigma}_{\max }$ and the corresponding optimal characteristic length $L_{\mathrm{opt}}$. With the value of $r$ given in Table 2, Fig. 6 shows the plots of $\bar{\sigma}_{\max }$ and $L_{\mathrm{opt}}$ for $0<d \leq 0.3$ The ranges of $\bar{\sigma}_{\max }$ and $L_{\mathrm{opt}}$ are obtained as $0.2760 \leq \bar{\sigma}_{\max } \leq 0.8016$ and $0.58 \leq L_{\mathrm{opt}} \leq 0.64$. It can be observed that the optimal value of $d$ is found to be 0.2 so that $d / r=1.0$, which results in $\bar{\sigma}_{\max }=0.8016$ at $L_{\mathrm{opt}}=0.62$.

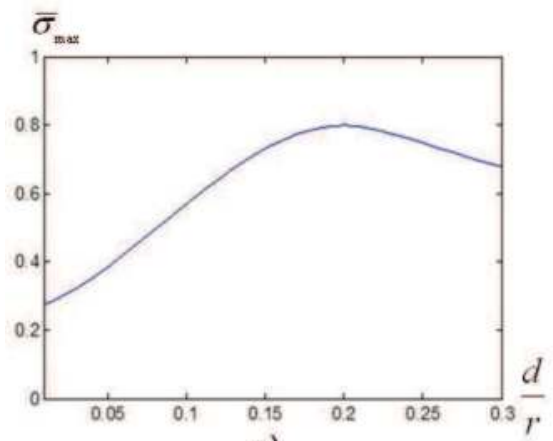

a)

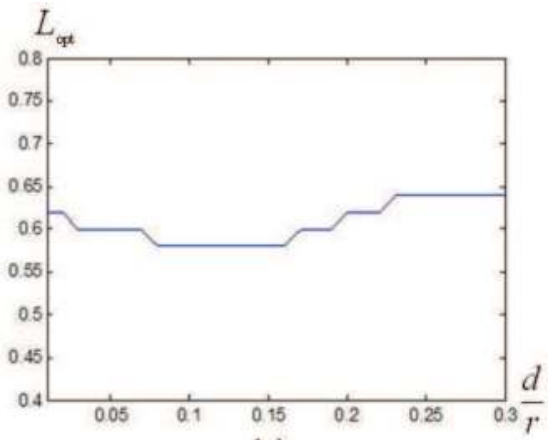

b)

Fig. 6. The plots of a) $\bar{\sigma}_{\max }$ and b) $L_{\text {opt }}$, for $0<d \leq 0.3$

\section{Conclusion}

In this paper, we presented the local and global isotropy analysis of a fully actuated caster wheeled omnidirectional mobile robot (COMR) with the steering link offset different from the wheel radius. First, based on the kinematic model, the necessary and sufficient isotropy conditions of a COMR were derived. Second, four different sets of all possible isotropic configurations were identified, along with the expressions for the isotropic steering link 
offset and the isotropic characteristic length. Third, using the local isotropy index, the number of the isotropic configurations and the role of the isotropic characteristic length were examined. Fourth, using the global isotropy index, the optimal characteristic length and the optimal steering link offset were determined for maximal global isotropy.

\section{Acknowledgement}

This work was supported by Hankuk University of Foreign Studies Research Fund of 2008, KOREA.

\section{References}

Holmberg, R. (2000). Design and Development for Powered-Caster Holonomic Mobile Robot. Ph. D. Thesis, Dept. of Mechanical Eng., Stanford University

Muir, P. F. \& Neuman, C. P. (1987). Kinematic modeling of wheeled mobile robots. J. of Robotic Systems, Vol. 4, No. 2, pp. 281-340

Campion, G.; Bastin, G. \& Novel, B. D`Andrea. (1996). Structural properties and classification of kinematic and dynamic models of wheeled mobile robots. IEEE Trans. Robotics and Automation, Vol. 12, No. 1, pp. 47-62

Kim, S. \& Kim, H. (2004). Isotropy analysis of caster wheeled omnidirectional mobile robot. Proc. IEEE Int. Conf. Robotics and Automation, pp. 3093-3098

Park, T.; Lee, J.; Yi, B.; Kim, W.; You, B. \& Oh, S. (2002). Optimal design and actuator sizing of redundantly actuated omni-directional mobile robots. Proc. IEEE Int. Conf. Robotics and Automation, pp. 732-737

Kim, S. \& Moon, B. (2005). Local and global isotropy of caster wheeled omnidirectional mobile robot. Proc. IEEE Int. Conf. Robotics and Automation, pp. 3457-3462

Oetomo, D.; Li, Y. P.; Ang Jr., M. H. \& Lim, C. W. (2005). Omnidirectional mobile robots with powered caster wheels: design guidelines from kinematic isotropy analysis. Proc. IEEE/RSJ Int. Conf. Intelligent Robots and Systems, pp. 3034-3039

Kim, S \& Jung, I. (2007). Systematic isotropy analysis of caster wheeled mobile robots with steering link offset different from wheel radius. Proc. IEEE Int. Conf. Robotics and Automation, pp. 2971-2976

McGhee, R. B. \& Frank, A. A. (1968). On the stability of quadruped creeping gaits. Mathematical Biosciences, Vol. 3, No. 3, pp. 331-351

Papadopoulos, E. G. \& Rey, D. A. (1988). A new measure of tipover stability margin for mobile manipulators. Proc. IEEE Int. Conf. Robotics and Automation, pp. 3111-3116

Strang, G. (1988). Linear Algebra and Its Applications. Saunders College Publishing

Saha, S. K.; Angeles, J. \& Darcovich, J. (1995). The design of kinematically isotropic rolling robots with omnidirectional wheels. Mechanism and Machine Theory, Vol. 30, No. 8, pp. 1127-1137 


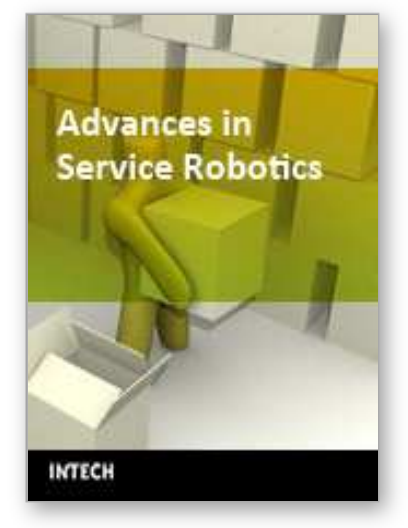

\author{
Advances in Service Robotics \\ Edited by Ho Seok Ahn
}

ISBN 978-953-7619-02-2

Hard cover, 342 pages

Publisher InTech

Published online 01, July, 2008

Published in print edition July, 2008

This book consists of 18 chapters about current research results of service robots. Topics covered include various kinds of service robots, development environments, architectures of service robots, Human-Robot Interaction, networks of service robots and basic researches such as SLAM, sensor network, etc. This book has some examples of the research activities on Service Robotics going on around the globe, but many chapters in this book concern advanced research on this area and cover interesting topics. Therefore I hope that all who read this book will find lots of helpful information and be interested in Service Robotics. I am really appreciative of all authors who have invested a great deal of time to write such interesting and high quality chapters.

\title{
How to reference
}

In order to correctly reference this scholarly work, feel free to copy and paste the following:

Sungbok Kim and Sanghyup Lee (2008). Local and Global Isotropy Analysis of Mobile Robots with Three Active Caster Wheels, Advances in Service Robotics, Ho Seok Ahn (Ed.), ISBN: 978-953-7619-02-2, InTech, Available from:

http://www.intechopen.com/books/advances_in_service_robotics/local_and_global_isotropy_analysis_of_mobil e_robots_with_three_active_caster_wheels

\section{INTECH}

open science | open minds

\author{
InTech Europe \\ University Campus STeP Ri \\ Slavka Krautzeka 83/A \\ 51000 Rijeka, Croatia \\ Phone: +385 (51) 770447 \\ Fax: +385 (51) 686166 \\ www.intechopen.com
}

\author{
InTech China \\ Unit 405, Office Block, Hotel Equatorial Shanghai \\ No.65, Yan An Road (West), Shanghai, 200040, China \\ 中国上海市延安西路65号上海国际贵都大饭店办公楼 405 单元 \\ Phone: +86-21-62489820 \\ Fax: $+86-21-62489821$
}


(C) 2008 The Author(s). Licensee IntechOpen. This chapter is distributed under the terms of the Creative Commons Attribution-NonCommercialShareAlike-3.0 License, which permits use, distribution and reproduction for non-commercial purposes, provided the original is properly cited and derivative works building on this content are distributed under the same license. 